\title{
Detection of pulsations in three subdwarf B stars ${ }^{\star \star \star \star}$
}

\author{
R. Østensen ${ }^{1}$, J.-E. Solheim ${ }^{1}$, U. Heber ${ }^{2}$, R. Silvotti ${ }^{3}$, S. Dreizler ${ }^{4}$, and H. Edelmann ${ }^{2}$ \\ 1 Institutt for Fysikk, Universitetet i Troms $\varnothing, 9037$ Tromsø, Norway \\ e-mail: janerik@phys.uit.no \\ 2 Dr. Remeis-Sternwarte, Astronomisches Institut der Universität Erlangen-Nürnberg, 96049 Bamberg, Germany \\ e-mail: ai03@sternwarte.uni-erlangen.de; edelmann@sternwarte.uni-erlangen.de \\ 3 Osservatorio Astronomico di Capodimonte, via Moiariello 16, 80131 Napoli, Italy \\ e-mail: silvotti@na.astro.it \\ 4 Institut für Astronomie und Astrophysik, Waldhäuser Straße 64, 72076 Tübingen, Germany \\ e-mail: dreizler@astro.uni-tuebingen.de
}

Received 10 August 2000 / Accepted 5 December 2000

\begin{abstract}
We report the detection of short period oscillations in the sdB stars HS $0815+4243$, HS $2149+0847$ and HS 2201+2610 from time-series photometry made at the Nordic Optical Telescope (NOT) from a sample of 31 candidates. Hence these three hot subdwarfs are new members of the EC 14026 class of pulsating sdB stars. One short period is detected for HS $0815+4243(P \sim 126 \mathrm{~s} ; A \sim 7 \mathrm{mma})$ and two short periods are seen for HS 2149+0847 ( $P \sim 142,159 \mathrm{~s} ; A \sim 11,7 \mathrm{mma})$, whereas the single oscillation detected for HS $2201+2610$ has a considerably longer period $(P \sim 350 \mathrm{~s} ; A \sim 11 \mathrm{mma})$. Our NLTE model atmosphere analysis of the timeaveraged optical spectra indicate that HS $0815+4243$ has $T_{\text {eff }}=33700 \mathrm{~K}$ and $\log g=5.95$, HS $2149+0847$ has $T_{\text {eff }}=35600 \mathrm{~K}$ and $\log g=5.9$, and HS $2201+2610$ has $T_{\text {eff }}=29300 \mathrm{~K}$ and $\log g=5.4$. This places the former two at the hot end and the latter at the cool end of the theoretical sdBV instability strip.
\end{abstract}

Key words. subdwarfs - stars: oscillations - stars: individual: HS 0815+4243 HS 2149+0847 HS 2201+2610

\section{Introduction}

The hot subdwarf B (sdB) stars are evolved low mass objects $\left(\sim 0.5 M_{\odot}\right)$ with helium burning cores, and are surrounded by a hydrogen surface layer too thin to sustain $\mathrm{H}$-shell burning ( $<1 \%$ by mass). Their high temperature and brightness place them close to the extreme horizontal branch (EHB) in the HR diagram. Their origin is still not well known; in the past they should have experienced a core He flash and substantial mass loss during or after the giant branch phase. After core He exhaustion they are expected not to evolve toward the asymptotic giant branch, but instead move left in the HR diagram, passing through parts of the subdwarf O population (Lemke et al. 1997),

Send offprint requests to: R. Østensen,

e-mail: roy@phys .uit.no

* Based on observations obtained at the Nordic Optical Telescope, operated on the island of La Palma jointly by Denmark, Finland, Iceland, Norway, and Sweden, in the Spanish Observatorio del Roque de los Muchachos of the Instituto de Astrofisica de Canarias.

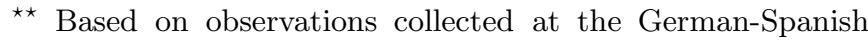
Astronomical Center, Calar Alto, operated by the Max-PlankInstitute für Astronomie Heidelberg jointly with the Spanish National Commission for Astronomy. and ultimately enter the white dwarf population as low mass WDs.

The recent discovery that many of them are multimode pulsators has opened a new attractive possibility of probing their interior using seismological tools. So far, data on 16 such pulsators have been published. 13 are summarised in the review by O'Donoghue et al. (1999), and three more have recently been published (Piccioni et al. 2000; Billères et al. 2000; Silvotti et al. 2000a). In this paper we present spectroscopy and photometry on another three, bringing the total known number of such stars up to 19. The sdB pulsators, also known as sdB Variables $=$ sdBVs or EC 14026 stars from the prototype (Kilkenny et al. 1997), have short pulsation periods ( 1-10 min) and low amplitudes ( 1-50 milli modulation amplitudes [mma]). About half of them are in binary systems with F-G stars. Charpinet et al. $(1996,1997)$ have shown that the pulsations of the sdB stars can be driven by an opacity bump associated with iron ionization. Both radial and nonradial modes are expected to have about the same range of frequencies, corresponding to what is observed. Similar period ranges have also been obtained by Kawaler (1999). 

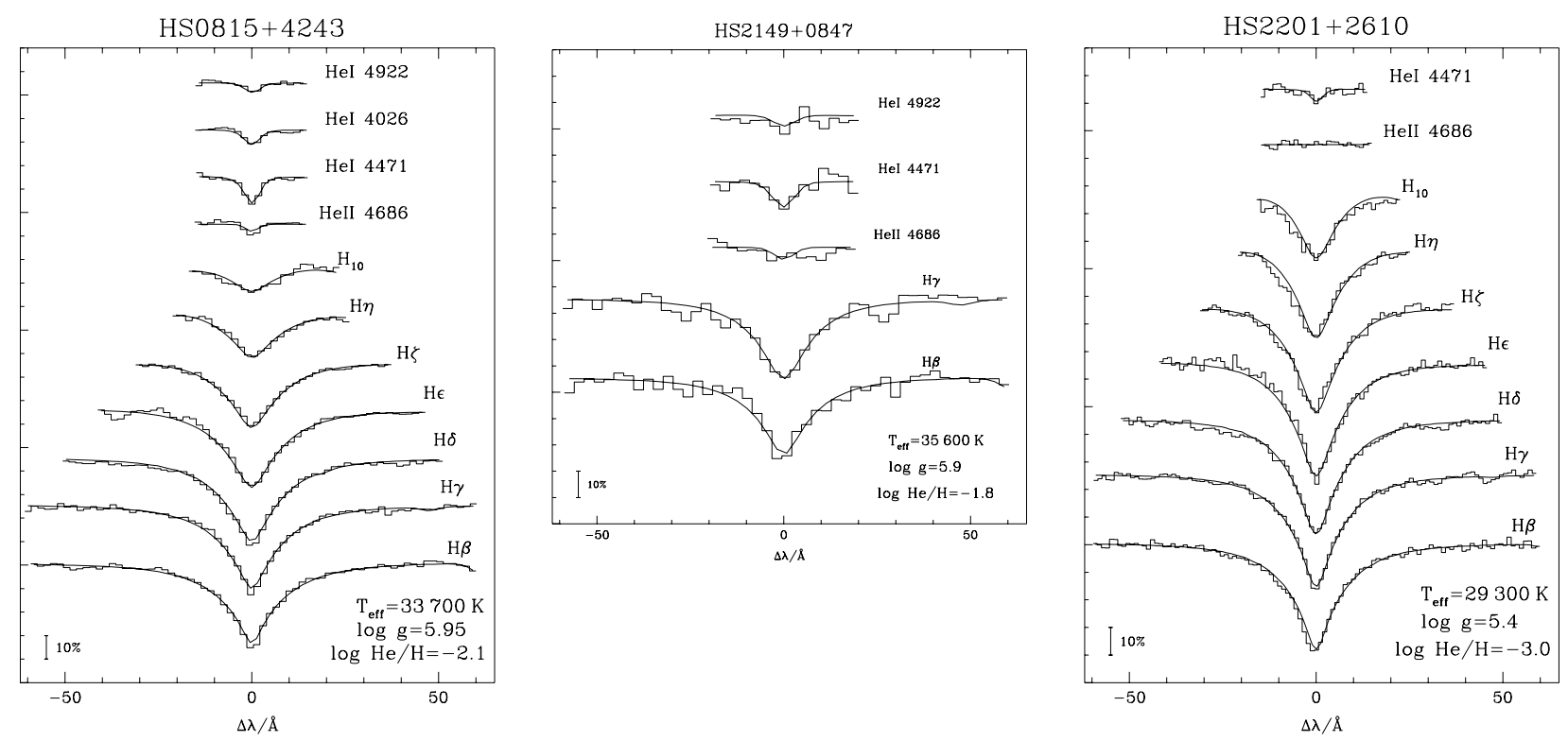

Fig. 1. Model fits to the Balmer and He line profiles in our time-averaged optical spectra of the three programme stars using NLTE model atmospheres (see text)

The three new pulsators reported in this paper have been taken from a list of candidates from the Palomar Green (Green et al. 1986) and Hamburg Schmidt surveys (Hagen et al. 1995) selected by follow-up spectroscopy on the basis of their atmospheric parameters (Heber et al. 1999; Edelmann et al. 2001). From this list, 13 objects were observed with continuous photoelectric photometry with the Nordic Optical Telescope (NOT) in July 1999 and one new pulsator, PG $1618+563 \mathrm{~B}$, was detected (Silvotti et al. 2000a). In October 1999, in a further run on the NOT, we used a CCD camera with our own controller software (Østensen \& Solheim 2000) to observe 31 additional candidates; three of these were identified as pulsators and the preliminarly results were shown by Silvotti et al. (2000b). A more detailed description of the technique used, and the upper limits to the pulsation amplitude of the sdBs in which we have not detected any pulsations above the $3 \sigma$ limit, will be reported in a subsequent paper when our search programme will be completed.

\section{Spectroscopy}

Medium resolution (4-6 $\AA$ ) spectra were obtained at the Calar Alto $3.5 \mathrm{~m}$ and $2.2 \mathrm{~m}$ telescopes equipped with the Twin spectrograph and the B\&C Cassegrain spectrograph, respectively; details of the observations are listed in Table 1. Besides the Balmer lines of hydrogen, He I lines are detected in all three stars whereas He II $4686 \AA$ is detected in HS $0815+4243$, but not found in the spectra of HS $2149+0847$ and HS $2201+2610$. No indication of cool companions to the sdB stars can be found in the spectra. The exposure times are much longer than their oscillation periods (see Sect. 3) and therefore the spectra are timeaveraged.
Table 1. Details of spectroscopic observations at the Calar Alto observatory. The last coloumn gives the size of the telescope and the detector used with its reciprocal dispersion in parantheses

\begin{tabular}{lcrcl}
\hline Object & $\begin{array}{c}\boldsymbol{B} \\
\mathbf{m a g}\end{array}$ & Date & $\begin{array}{c}\text { Exp } \\
\mathbf{s}\end{array}$ & $\begin{array}{l}\text { Detector } \\
\mathbf{m}(\boldsymbol{\AA} / \mathbf{m m})\end{array}$ \\
\hline HS 0815+4243 & 16.1 & 25.1 .95 & 3600 & $3.5 /$ Twin $(72)$ \\
HS $2149+0847$ & 16.5 & 5.9 .93 & 4000 & $2.2 /$ Cas. $(120)$ \\
HS $2201+2610$ & 13.6 & 31.8 .97 & 1800 & $3.5 /$ Twin $(72)$ \\
\hline
\end{tabular}

A grid of synthetic spectra derived from $\mathrm{H}-\mathrm{He}$ line blanketed NLTE model atmospheres (Napiwotzki 1997) was matched to the data to simultaneously determine effective temperature, gravity and He abundance (Heber et al. 1999). The results are shown in Fig. 1, and described below for each star separately.

\subsection{HS $0815+4243$}

HS 0815+4243, hereafter HS 0815, has independently been classified as a "normal or horizontal branch star" by the Kiso survey (KUV 08159+4243, Wegner \& Mc Mahon 1986) and as a faint blue star by the first Byurakan spectral sky survey (FBS 0815+427= FBS B 251, Abrahamian et al. 1990).

Its $V$-magnitude as listed by Wegner \& Mc Mahon (1986, $V=16.3 \mathrm{mag}$ ) is consistent with the $B$-magnitude estimated from the Hamburg Schmidt plates $(B=$ $16.1 \mathrm{mag})$.

The available spectra are of excellent quality and therfore the atmospheric parameters can be derived with very small formal fitting errors, i.e. $\Delta\left(T_{\text {eff }}\right)= \pm 100 \mathrm{~K}$, 


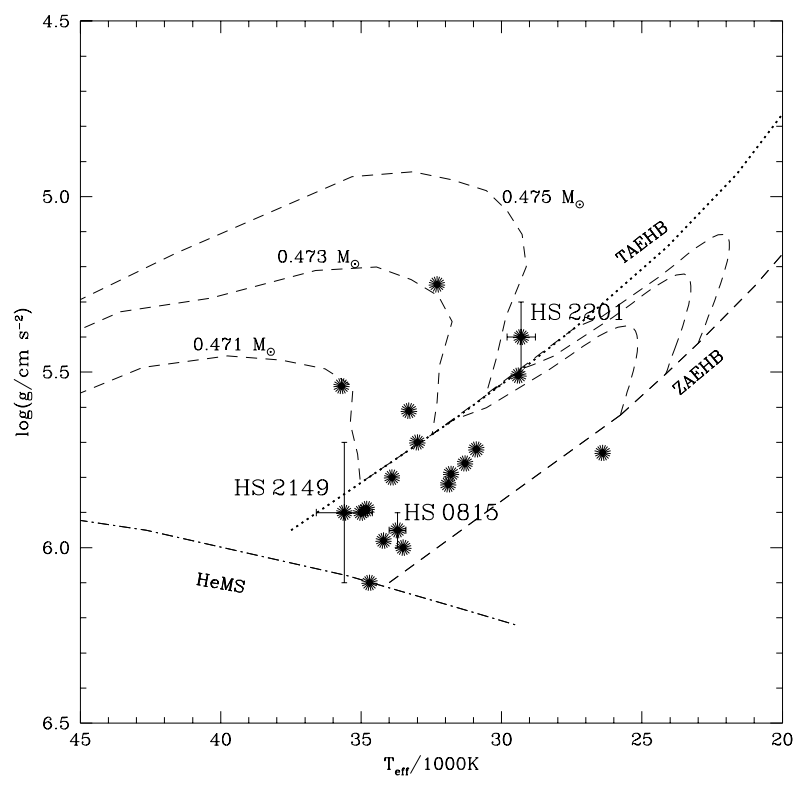

Fig. 2. Comparison of the three newly discovered sdBVs to previously known ones in the $\left(T_{\text {eff }}, \log g\right)$ plane. The position of the Zero Age Extreme Horizontal Branch (ZAEHB), the Terminal Age Extreme Horizontal Branch (TAEHB), the Helium Main Sequence (HeMS) and evolutionary tracks for EHB stars (Dorman et al. 1995) are also shown

$\Delta(\log g)= \pm 0.02$ dex and $\Delta(\log (N(\mathrm{He}) / N(\mathrm{H})))= \pm 0.03$. It is worthwhile to note that the $\mathrm{HeI} / \mathrm{HeII}$ ionization equilibrium is well matched along with the Balmer line profiles. However, systematic errors, which can only be quantified by repeated observations, contribute significantly to the error budget. Thus, we adopt the following parameters: $T_{\text {eff }}=33700 \pm 300 \mathrm{~K}, \log g=5.95 \pm 0.05$, $\log (N(\mathrm{He}) / N(\mathrm{H}))=-2.1 \pm 0.1$.

\subsection{HS $2149+0847$}

For this star, hereafter HS 2149, we have found no additional published information. Our spectra are of poorer quality and cover only $\mathrm{H} \beta$ to $\mathrm{H} \gamma$. Hence the formal fitting errors are larger than for the two other stars, i.e. $\Delta\left(T_{\text {eff }}\right)= \pm 500 \mathrm{~K}, \Delta(\log g)= \pm 0.1$ dex and $\Delta(\log (N(\mathrm{He}) / N(\mathrm{H})))= \pm 0.05$. Again considering systematic errors we adopt: $T_{\text {eff }}=35600 \pm 1000 \mathrm{~K}, \log g=$ $5.9 \pm 0.2, \log (N(\mathrm{He}) / N(\mathrm{H}))=-1.8 \pm 0.2$.

\subsection{HS $2201+2610$}

The sdBV star HS $2201+2610$, hereafter HS 2201, is one of the brightest in our sample $(B=13.6$, estimated from the Hamburg Schmidt plates), but nevertheless we have found no additional information in the SIMBAD database. Our spectroscopic data are of good quality and the formal fitting errors are small: $\Delta\left(T_{\text {eff }}\right)= \pm 200 \mathrm{~K}, \Delta(\log g)=$ \pm 0.03 dex and $\Delta(\log (N(\mathrm{He}) / N(\mathrm{H})))= \pm 0.15$. Again considering systematic errors we adopt: $T_{\text {eff }}=29300 \pm 500 \mathrm{~K}$, $\log g=5.4 \pm 0.1, \log (N(\mathrm{He}) / N(\mathrm{H}))=-3.0 \pm 0.3$.
Table 2. Time-series photometry

\begin{tabular}{|c|c|c|c|c|}
\hline Object & $\begin{array}{c}\text { Date } \\
(1999)\end{array}$ & Observers & $\begin{array}{l}\text { Start } \\
\text { (UT) }\end{array}$ & $\begin{array}{c}\text { Length } \\
(\mathrm{s})\end{array}$ \\
\hline \multirow[t]{3}{*}{ HS $0815+4243$} & Oct. 16 & $\mathrm{R} \varnothing, \mathrm{JES}$ & $05: 23$ & 3880 \\
\hline & Oct. 17 & RØ, JES & $05: 59$ & 2020 \\
\hline & Oct. 18 & RØ, JES & 05:04 & 5800 \\
\hline \multirow[t]{2}{*}{ HS $2149+0847$} & Oct. 17 & RØ, JES & $23: 25$ & 4020 \\
\hline & Oct. 18 & RØ, JES & $22: 13$ & 3620 \\
\hline HS $2201+2610$ & Oct. 18 & RØ, JES & $23: 20$ & 2660 \\
\hline
\end{tabular}

\subsection{Discussion}

The determined temperatures, gravities and helium abundances are typical for pulsating sdB stars, as can be seen from a comparison to known sdBVs (Fig. 2).

HS 2201 has the lowest effective temperature, gravity and helium abundance of the three stars analysed here. It can be considered as a spectroscopic twin to the sdBV star Feige 48, because its atmospheric parameters agree with that of the latter (Heber et al. 2000) to within error limits.

HS 2149 and PB 8783 (Koen et al. 1997) are the hottest known sdBV stars, and might define the blue edge of the instability strip.

HS 0815 has similar effective temperature and gravity as the sdBV stars EC 10228-0905 (Stobie et al. 1997) and PG 1047+003 (O'Donoghue et al. 1998) and lies in the region of the $\left(T_{\text {eff }}, \log g\right)$ diagram where most sdBV stars are found (see Fig. 2).

\section{Time-series photometry}

The observations were done with the NOT High Resolution Adaptive Camera (HiRAC) with a Loral, Lesser thinned, $2048 \times 2048$ CCD chip, and modified with our own control software to be able to observe in highspeed multi-windowing mode.

The sky area available for locating a reference star is limited to the area of the chip: $\sim 3.7 \times 3.7 \operatorname{arcmin}^{2}$.

\subsection{Observations and reductions}

Table 2 contains the information related to the time-series observations. We observed the stars in windowed mode using two or more reference stars for constructing the relative light curve. All the observations were made with the HiRAC $B$-band filter, and with a $20 \mathrm{~s}$ cycle time. The actual exposure times varied as we started out with five $64 \times 64$ pixel windows (target, two reference stars and two sky fields) and an exposure time of $14.7 \mathrm{~s}$, and later changed this to six $42 \times 42$ pixel windows (one additional reference star) and an exposure time of $15.8 \mathrm{~s}$.

The data were reduced on-line using the Real Time Photometry (RTP) program developed by one of us (R. Østensen) as part of his Ph.D-project. This program 


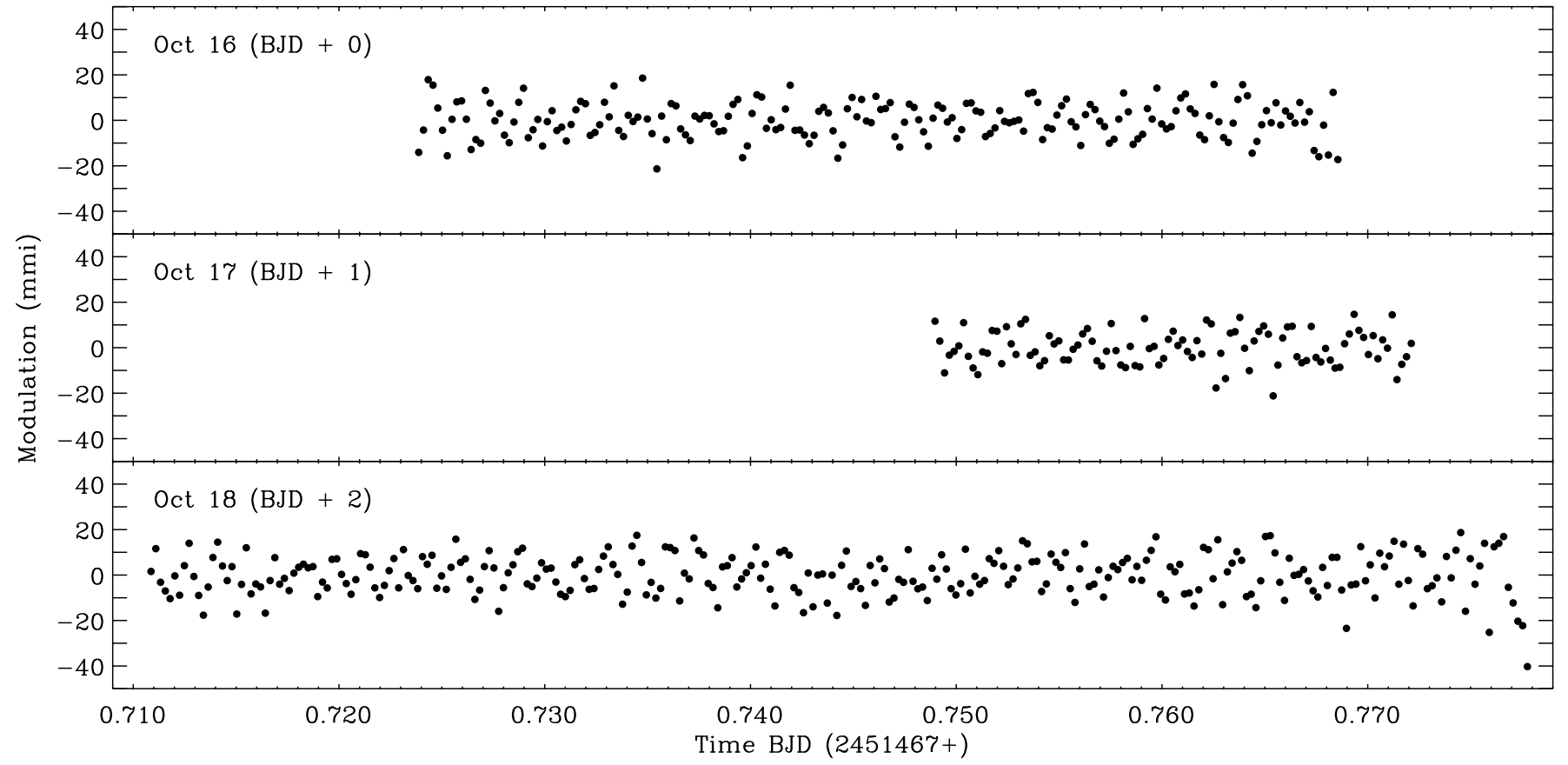

Fig. 3. Light curves for HS $0815+4243$

allows us to detect periodic variable stars with small amplitudes from CCD data in real time. Usually we could say in $\sim 30$ min that a target star was not pulsating, and then move on to another star. Our graphical interface used to mark the target, the reference stars and sky fields from a full CCD image, permits us to set up a new observation in a very short time $(\sim 5 \mathrm{~min})$. Thus, we were able to investigate more than twice as many targets per night than we could during our previous run of July 1999, when a classical three-channel photoelectric photometer was used.

The RTP program automatically converts all data to the correct bias level, performs appropriate flat-fielding and computes the sum of ADU counts for the target and reference stars within a circular aperture. The sky level is computed from the pixels in the sky field using a three sigma median clipping algorithm to reject cosmic rays and bad pixels. This sky value is then subtracted from the counts of each star, and the differential photometry between target and reference stars is computed. RTP presents all these light curves (raw data, sky subtracted data and differential photometry) propagating in real time as the windowed frames are acquired from the CCD camera. At any point a Fourier Transform (FT) can be computed from the data acquired so far.

The CCD multi-windowed photometry method also gives us opportunity to reprocess the data, applying different aperture sizes and correction schemes. One method recently implemented, that proved to be especially valuable in this case, is the moving aperture photometry (MAP) technique. MAP computes the centre of the target in each frame and recenters the aperture correspondingly. In this way it is possible to correct for small tracking errors on the order of 0.05 arcseconds, allowing the use of very small
Table 3. Pulsation frequencies and amplitudes

\begin{tabular}{cccccc}
\hline Object & $\begin{array}{c}\text { Date } \\
(\mathbf{1 9 9 9})\end{array}$ & $\begin{array}{c}\boldsymbol{F}_{\mathbf{1}} \\
(\mathbf{m H z})\end{array}$ & $\begin{array}{c}\boldsymbol{F}_{\mathbf{2}} \\
\left(\mathbf{m} \boldsymbol{\boldsymbol { A } _ { \mathbf { 1 } }}\right.\end{array}$ & $\begin{array}{c}\boldsymbol{A}_{\mathbf{2}} \\
(\mathbf{m a})\end{array}$ \\
\hline HS 0815+4243 & Oct. 16 & 7.92 & & 7.8 & \\
& Oct. 17 & 7.92 & & 5.9 & \\
& Oct. 18 & 7.92 & & 7.3 & \\
HS 2149+0847 & Oct. 17 & 7.07 & 6.29 & 11.2 & 7.1 \\
& Oct. 18 & 7.08 & 6.26 & 11.8 & 6.7 \\
HS 2201+2610 & Oct. 18 & 2.85 & & 11.2 & \\
\hline
\end{tabular}

apertures, which significantly reduce the noise contribution from the sky background.

Finally, all the data were reduced again with a more accurate procedure (compensation of long time-scale trends, improved flat-fielding and extinction corrections). The resulting light-curves are shown in Figs. 3, 5 and 7, and are discussed below for each of the objects. The light curves show differential photometry between the target and the best combination of the available reference stars.

The optimal aperture for each run was selected after processing all data sets with apertures of a wide range of diameters and choosing the one that gave the best signalto-noise ratio in the amplitude spectrum (using the amplitude of the primary peak for the signal and the mean of the amplitude spectrum outside the pulsation range for the noise). The apertures we use range between 22 and 30 pixels, which corresponds to 2.4 and 3.3 arcsec.

Figures 4, 6 and 8 show the FTs of the light curves. The significant peaks detected in these amplitude spectra are listed in Table 3. 


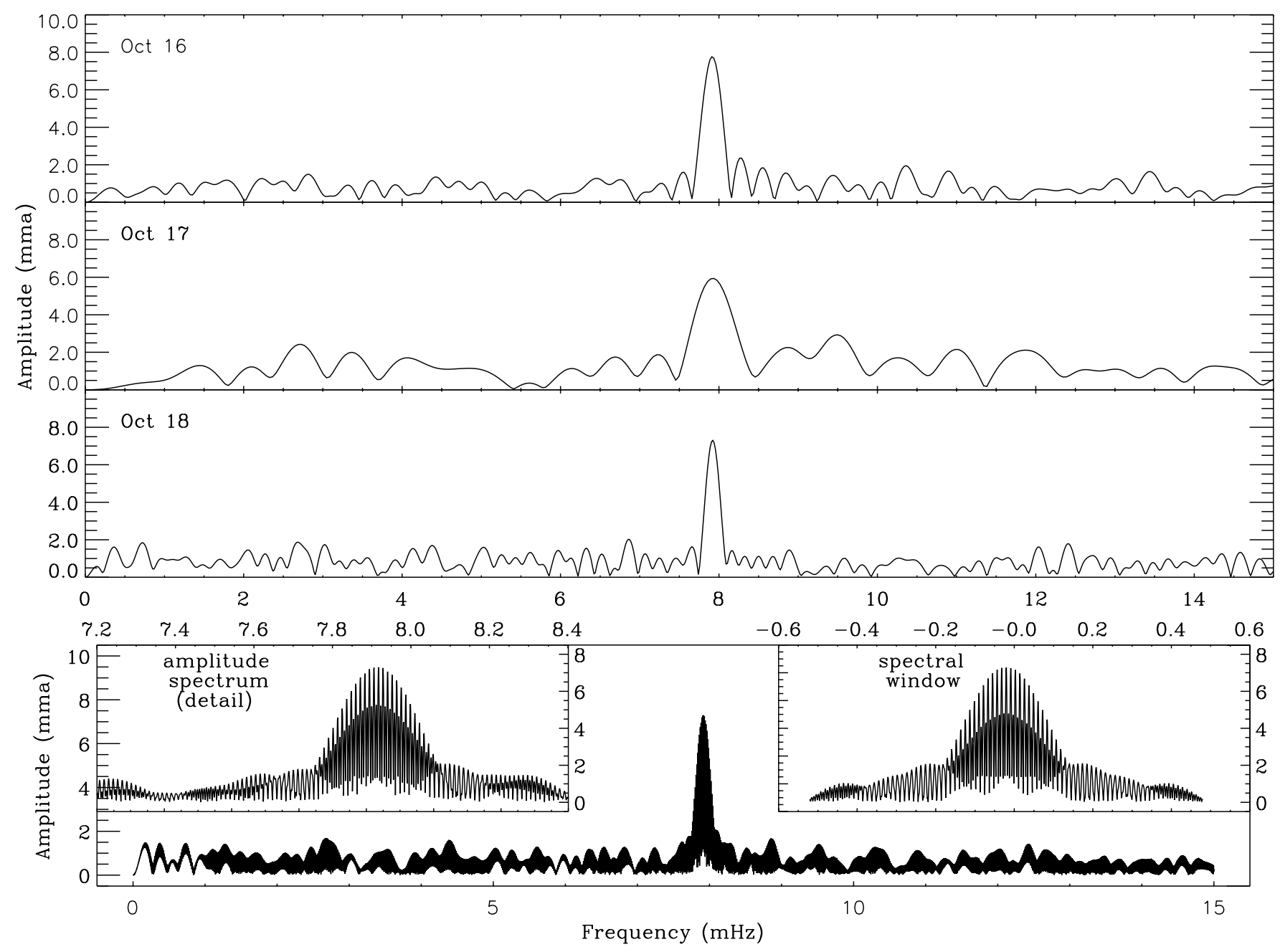

Fig. 4. Amplitude spectra for HS $0815+4243$. The three upper panels show the individual spectra for the three nights, and the lower panel shows the spectrum of the three consecutive nights taken together. The two inset panels represent the same spectrum with a larger scale (left) and its spectral window (right)

\subsection{Data analysis and results}

\subsubsection{HS $0815+4243$}

All the three amplitude spectra show a dominant peak at $7.92 \mathrm{mHz}$ with amplitude of about $7 \mathrm{mma}$. The run on the second night was started late in the night, and thus was too short to get a fair resolution in the temporal spectrum. An FT of the window function (not shown) indicates that the small peaks close to the main peak most likely are artifacts of the spectral window. A longer run would be required to resolve the temporal spectrum of this star. The average noise level is below $0.8 \mathrm{mma}$ for both the first and third run, and below $1.1 \mathrm{mma}$ for the second run, when the frequency range 7.0 to $8.5 \mathrm{mHz}$ is disregarded.

In the bottom panel of Fig. 4, the combined FT of the three nights is shown. No indications of other than the main frequency, seen in the three individual runs, is indicated at this level of resolution. The average noise level in the combined spectrum is below $0.6 \mathrm{mma}$.

The extinction corrected light curves indicate an average magnitude $B=16.0$ for all three nights, in good agreement with the magnitude estimated from the photographic plates.

\subsubsection{HS $2149+0847$}

This object was identified as a variable on October 17, and clearly shows two pulsation frequencies at 7.1 and $6.3 \mathrm{mHz}$, with amplitudes of about 7 and $11 \mathrm{mma}$, respectively. There is an indication of a third frequency between these at about $6.7 \mathrm{mHz}$, showing an amplitude of 2.1 and $3.4 \mathrm{mma}$ in the first and second night respectively (Fig. 6). The lower panel of Fig. 6 shows the FT of the two nights together. Comparing the inset part of the temporal spectrum to the inset spectral window, it is clear that the peak between the two main frequencies is an artifact.

The mean noise level is about $1.2 \mathrm{mma}$ in the first night and $0.9 \mathrm{mma}$ in the second when the frequency region with the main peaks (between 6.0 and $8.0 \mathrm{mHz}$ ) is disregarded. In the combined spectrum the average noise level is about $0.8 \mathrm{mma}$. 


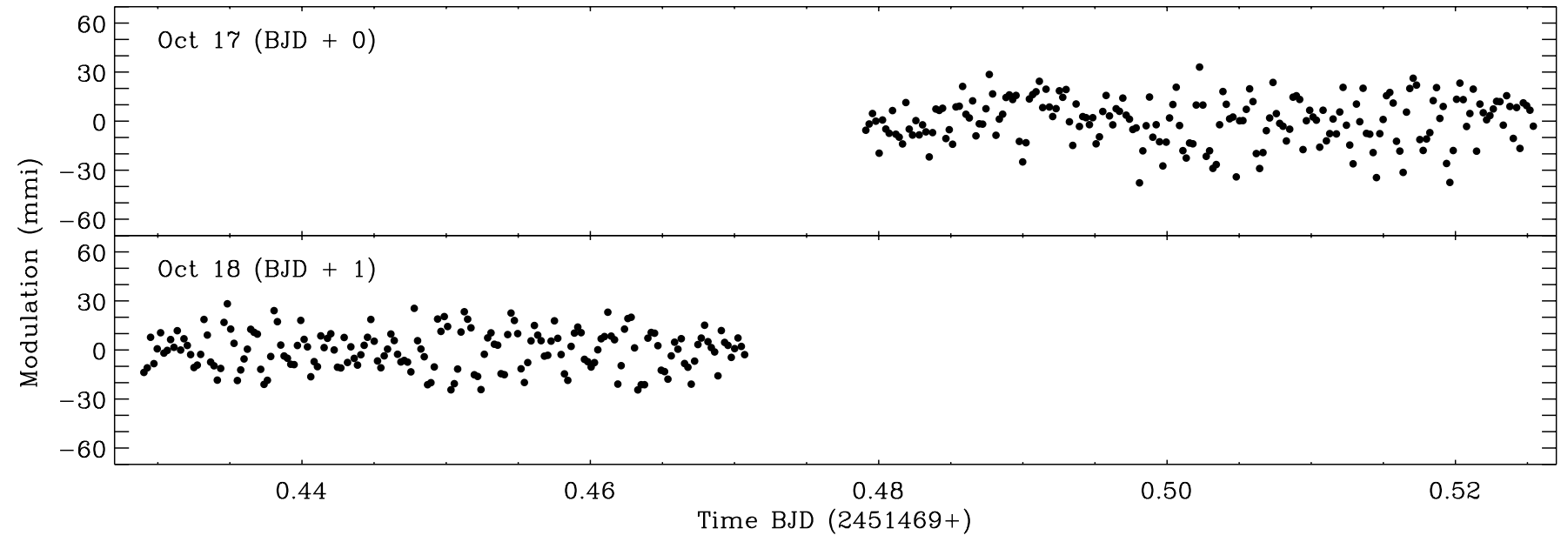

Fig. 5. Light curves for HS $2149+0847$

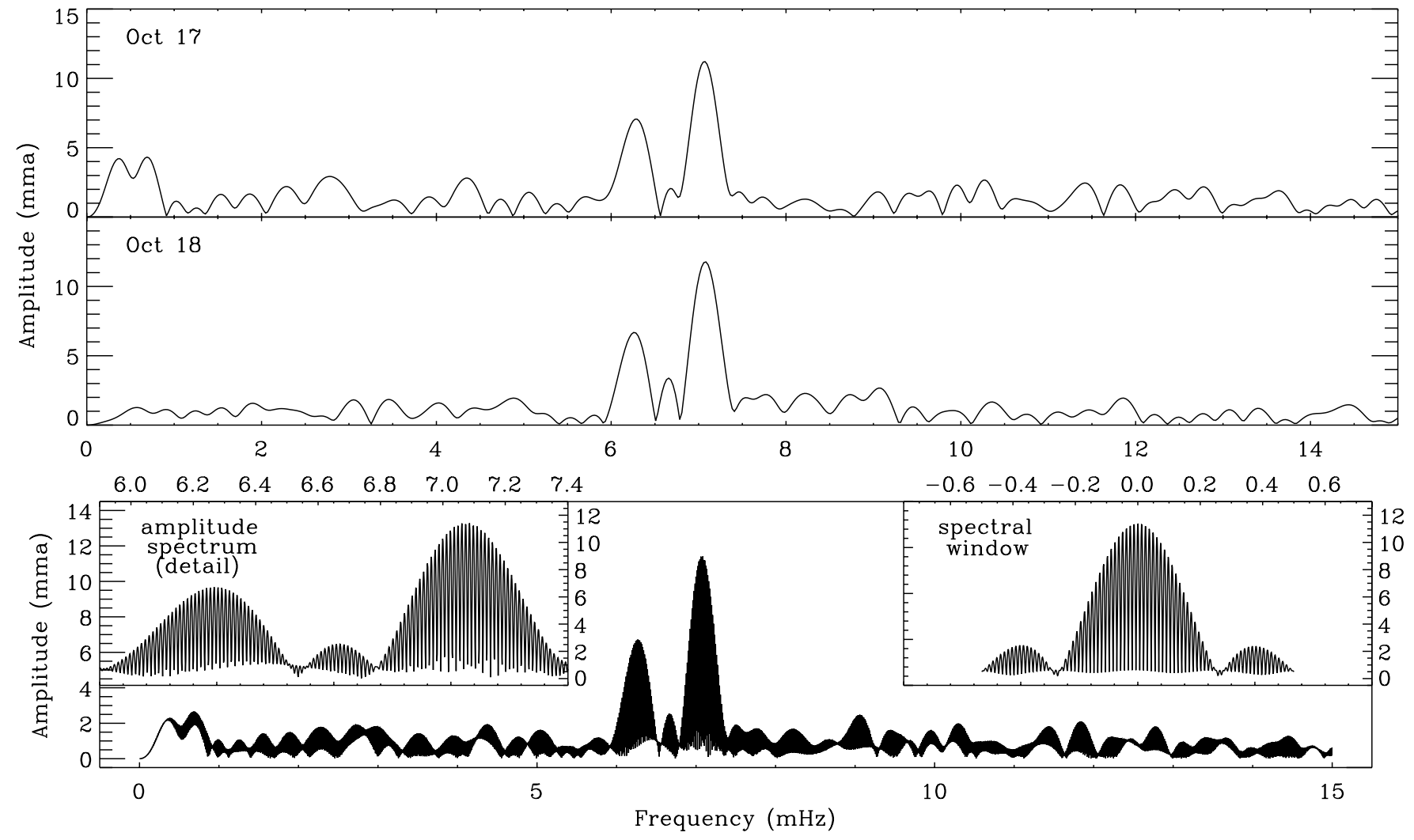

Fig. 6. Amplitude spectra for HS $2149+0847$. The upper panels show the spectra for each night, while the lower shows the spectrum from both runs taken together. The insets give the same spectrum magnified (left) and its spectral window (right)

In the light curves we can clearly see the beat period of about $1200 \mathrm{~s}$ (Fig. 5). The extinction corrected photometry gives us a magnitude $B=16.4$ on both nights for this target.

\subsubsection{HS $2201+2610$}

This object was discovered to be variable during the last observing night, and even after only a few minutes of observations it was clear that HS 2201 is a pulsator. The frequency of the pulsation is $2.85 \mathrm{mHz}$, with an amplitude of $11 \mathrm{mma}$. Since the run was only $40 \mathrm{~min}$ long, the resolution of $0.4 \mathrm{mHz}$ may hide considerable fine structure, as it can be seen in Fig. 8. The noise level in the higher frequencies (above $5 \mathrm{mHz}$ ) is below $0.4 \mathrm{mma}$.

The extinction corrected light curve gives $B=14.3$ for this target, which is not in very good agreement with the magnitude estimated from the original Hamburg Schmidt 


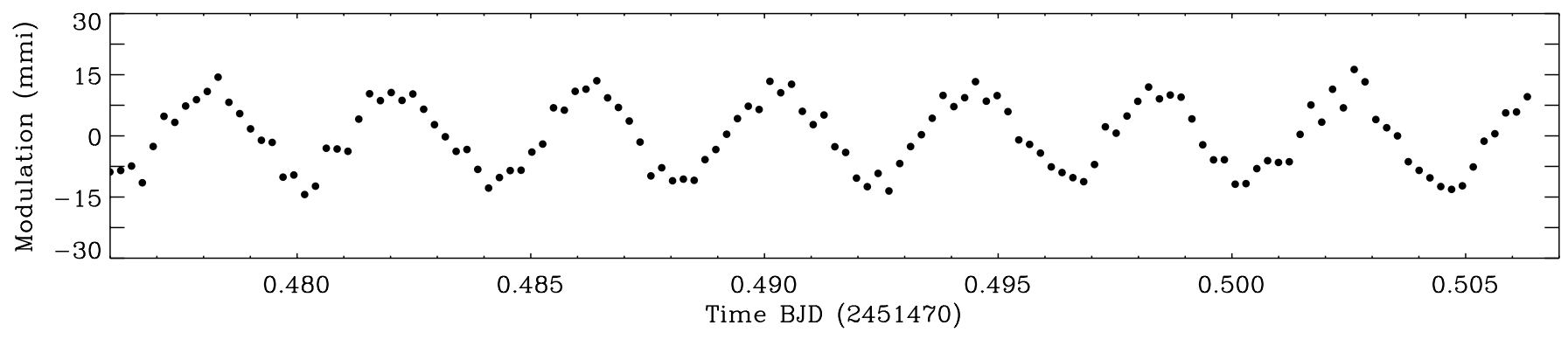

Fig. 7. Light curve for HS $2201+2610$

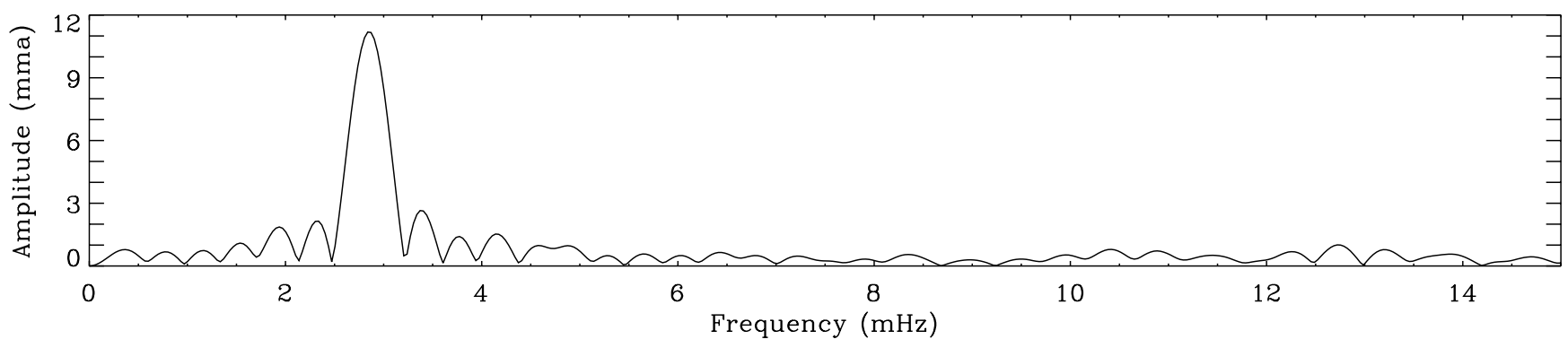

Fig. 8. Amplitude spectrum for HS 2201+2610

plates. Since we had some cirrus throughout the night we cannot say with certainty which estimate is the most correct one.

\section{Summary and discussion}

The three sdB stars discovered to be variable, which are located well inside the region of the $\left(T_{\text {eff }}, \log g\right)$ plane where the sdB pulsators are expected, have quite simple temporal spectra, as far as we can judge from our short light curves.

HS 2201 has almost the same $T_{\text {eff }}, \log g$ and pulsation period as Feige 48, which shows at least 4 periods with variable amplitudes (Koen et al. 1998). For Feige 48 the strongest pulsation at $2.87 \mathrm{mHz}$ can vary between 4.4 and $13 \mathrm{mma}$ from one night to the next. Although HS 2201 appears to have only a single pulsation, the frequency of $2.85 \mathrm{mHz}$ and amplitude of $11 \mathrm{mma}$ matches the main pulsation of Feige 48 perfectly, so the connection is quite clear. Since we investigated HS 2201 only during one night, we had no opportunity to check if its amplitude is variable. The low resolution of the FT does not rule out multiple periods close to the main period.

The other two hot pulsators near $T_{\text {eff }}=35000 \mathrm{~K}$, are placed just on (for HS 2149) or below (for HS 0815) the locus of the fundamental mode, in the Period/log $g$ graph (Fontaine et al. 1998), indicating that the pulsations we observed either are associated with the fundamental mode or strong $p$-modes.

Our first variable star search campaign using the windowed CCD photometry technique proves that this system is excellent for this kind of work, and gives several improvements compared to the standard photoelectric technique. With a medium size telescope we can detect low amplitude pulsators in runs only about $30 \mathrm{~min}$ long, and get reliable identification of the primary modes in one hour.

Acknowledgements. This research was supported by the Norwegian Research Council. R.S. acknowledges support by the italian research foundation MURST under project "Stellar Evolution" (coordinator V. Castellani). H.E. acknowledges financial support by the german research foundation DFG under grant He 1354/30-1. The authors also wishes to thank the staff at the NOT for good support. S. D. and U. H. thank D. Engels, H. Hagen and D. Reimers (Hamburg) for a fruitful collaboration over many years.

This research has made use of the SIMBAD database, operated at CDS, Strasbourg, France.

\section{References}

Abrahamian, H. V., Lipovetsky, V. A., Mkaelian, A. M., \& Stephanian, J. A. 1990, Afz, 33, 345

Billères, M., Fontaine, G., Brassard, P., et al. 2000, ApJ, 530, 441

Charpinet, S., Fontaine, G., Brassard, P., \& Dorman, B. 1996, ApJ, 471, L106

Charpinet, S., Fontaine, G., Brassard, P., et al. 1997, ApJ, 483, L123

Dorman, B., O'Connell, R. W., \& Rood, R. T. 1995, ApJ, 442, 105

Edelmann, H., Heber, U., Lemke, M., et al. 2001, A\&A, in preparation

Fontaine, G., Charpinet, S., Brassard, P., et al. 1998, New Eyes to See Inside the Sun and Stars, IAUS, 185, 367

Green, R. F., Schmidt, M., \& Liebert, J. 1986, ApJS, 61, 305

Hagen, H.-J., Groote, D., Engels, D., \& Reimers, D. 1995, A\&AS, 111, 195

Heber, U., Edelmann, H., Lemke, M., Napiwotzki, R., \& Engels, D. 1999, PASPC, 169, 551 
Heber, U., Reid, I. N., \& Werner, K. 2000, in Proc. of the 5th Whole Earth Telescope Workshop, ed. G. Vauclair, \& E. G. Meištas, Baltic Astron., 9, 171

Kawaler, S. D. 1999, in Proc. of the 11th European Workshop on White Dwarfs, ed. J.-E. Solheim, \& E. G. Meištas, ASP Conf. Ser., 169, 158

Kilkenny, D., Koen, C., O’Donoghue, D., \& Stobie, R. S. 1997, MNRAS, 285, 640

Koen, C., Kilkenny, D., O'Donoghue, D., Van Wyk, F., \& Stobie, R. S. 1997, MNRAS, 285, 645

Koen, C., O'Donoghue, D., Pollacco, D. L., \& Nitta, A. 1998, MNRAS, 300, 1105

Lemke, M., Heber, U., Napiwotzki, R., Dreizler, S., \& Engels, D. 1997, in The third conference on Faint Blue Stars, ed. A. G. D. Philip, J. W. Liebert, \& R. A. Saffer (L. Davis press), 375

Napiwotzki, R. 1997, A\&A, 322, 256

O'Donoghue, D., Koen, C., Lynas-Gray, A. E., Kilkenny, D.,
\& Van Wyk, F. 1998, MNRAS, 296, 306

O’Donoghue, D., Koen, C., Kilkenny, D., \& Stobie, R. S. 1999, in Proc. of the 11th European Workshop on White Dwarfs, ed. J.-E. Solheim, \& E. G. Meištas, ASP Conf. Ser., 169, 149

Østensen, R., \& Solheim, J.-E. 2000, in Proc. of the 5th Whole Earth Telescope Workshop, ed. G. Vauclair, \& E. G. Meištas, Baltic Astron., 9, 411

Piccioni, A., Bartolini, C., Bernabel, S., et al. 2000, A\&A, 354, L13

Silvotti, R., Solheim, J.-E., Gonzalez-Perez, J. M. et al. 2000a, A\&A, 359, 1068

Silvotti, R., Solheim, J.-E., Heber, U., et al. 2000b, in Proc. of the 12th European Worskhop on White Dwarfs, ASP Conf. Ser., in press

Stobie, S., Kawaler, S. D., Kilkenny, D., O'Donoghue, D., \& Koen, C. 1997, MNRAS, 285, 651

Wegner, G., \& Mc Mahon, R. G. 1986, AJ, 91, 139 